\title{
Relationship between the extent of resection and the survival of patients with low-grade gliomas: a systematic review and meta-analysis
}

\author{
Liang $\mathrm{Xia}^{1 \dagger}$, Chenyan Fang ${ }^{3 \dagger}$, Gao Chen ${ }^{2^{*}}$ and Caixing Sun ${ }^{1 *}$
}

\begin{abstract}
Background: Surgical resection is necessary to conduct a pathological biopsy and to achieve a reduction of intracranial pressure in low-grade gliomas patients. This study aimed to determine whether a greater extent of resection would increase the overall 5-year and 10-year survival of patients with low-grade gliomas.

Methods: The studies addressing relationship between the extent of resection and the prognosis of low-grade gliomas updated until March 2017 were systematically searched in two databases (Pubmed and EMBASE). The relationships among categorical variables were analyzed using an odds ratio (OR) and a95\% confidence interval (Cl). Significance was established using $\mathrm{Cls}$ at a level of $95 \%$ or $P<0.05$. Funnel plot was used to detect the publication bias.

Results: Twenty articles (a total of 2128 patients) were identified. The meta-analysis showed that the 5 -year (Odds ratio (OR) , 3.90;95\% Confidence Interval (Cl), 2.79 5.45; $P<0.01 ; Z=7.95)$ and 10-year OS $(\mathrm{OR}, 7.91 ; 95 \% \mathrm{Cl}, 5.12 \sim 12.22 ; P<0.01 ; Z=9$. 33) associated with gross total resection (GTR) were higher than those associated with subtotal resection (STR). Similarly, as compared with biopsy(BX), the 5-year and 10-year OS were higher after either GTR (5-year: OR, 5.43; 95\%Cl, 3.57 8.26; $P<0$. $01 ; Z=Z=7.9 ; 10$-year: $O R, 10.17 ; 95 \% \mathrm{Cl}, 4.02 \sim 25.71 ; P<0.00001 ; Z=4.9)$ or STR (5-year: OR, 2.59; 95\%Cl, 1.81 - 3.71; $P<0$. $00001 ; Z=5.19 ; 10$-year: $O R, 2.21 ; 95 \% C l, 1.164 .25 ; P=0.02 ; Z=2.39$ ).

Conclusions: Our research found that a greater extent of resection could significantly increase the OS of patients with low-grade gliomas.
\end{abstract}

Keywords: Extent of resection, Low-grade Gliomas, Prognosis

\section{Background}

Low-grade gliomas include astrocytoma, oligodendrogliomaand oligoastrocytoma of WHO gradeI-II [1]. The incidence of low-grade gliomas is significantly lower than that of high-grade glioblastomas of all primary intracranial tumors [2]. The epidemiological features, clinical manifestations, proliferation rates, mitotic counts, as well as angiogenesis and genetic features of low-grade gliomas are different from those

\footnotetext{
* Correspondence: d-chengao@zju.edu.cn; 2226124552@qq.com

${ }^{\dagger}$ Equal contributors

${ }^{2}$ Department of Neurosurgery, The second affiliated hospital of Zhejiang University, Hangzhou, Zhejiang Province 310000, China

'Department of Neurosurgery, Zhejiang Cancer Hospital, 1 ban shan east Road, Hangzhou, Zhejiang Province 310022, China

Full list of author information is available at the end of the article
}

of high-grade gliomas [3]. Low-grade gliomas have a better prognosis than high-grade gliomas. The established risk factors influencing the prognosis of high-grade gliomas include IDH mutation, age, KPS score, and the extent of resection [4]. However, the prognostic factors of low-grade gliomas are not fully elucidated yet. So far, only IDH mutation, KPS score, age and the pathological type are recognized as factors related to the prognosis of low-grade gliomas [5], the effect on prognosis of extent of resection of lowgrade gliomas has not been systematically evaluated.

Many neurosurgeons recommend performing the greatest extent of resection safely possible for both highgrade and low-grade gliomas. In the past, the available surgical techniques might make it difficult to access 
gliomas in deep locations or in the brain functional areas [6]. However, with the use of neuronavigator and intraoperative MRI, many difficulties in accessing the tumors in challenging locations been solved [7]. High-grade gliomas have a higher incidence and their median survival ranges from 1 to 2 years [4]. A large number of researches have tried to elucidate the association between the extent of resection and the prognosis of high-grade gliomas. There are explicit first-level evidences indicating that a gross total resection (GTR) can significantly increase the overall survival (OS) and progression-free survival (PFS) of patients as compared with a subtotal resection (STR) or biopsy(BX) [8]. In contrast, low-grade gliomas have a lower incidence and a better prognosis. The medial survival of patients with low-grade gliomas is 5 to 10 years [3]. However, there are much fewer cases of low-grade gliomas and relevant clinical trials are hindered by long trial durations and ethical principles. At present, few researches have been focused on the relationship between the extent of resection and the prognosis of low-grade gliomas. Therefore, no randomized trials or first-level evidence have demonstrated explicitly the relationship between the extent of resection and prognosis.

It remains controversial whether a greater extent of resection can increase the OS and PFS of patients with low-grade gliomas. We performed a meta-analysis to prove the relationship between the extent of resection and the prognosis of patients with low-grade gliomas, then provide a basis for the development of evidencebased medicines in low-grade gliomas.

\section{Methods}

\section{Search strategy and study selection}

Using the PICO strategy, PubMed and EMBASE were searched for publications up to March 2017. The keywords chosen for the search included low-grade glioma (WHO gradeI-II), extent of resection, resection, biopsy and survival. The scope of search was expanded by combining the keywords with non-keywords according to the restriction of the English-language. Auxiliary search techniques such as keywords expansion were used to increase the recall rate. Detailed search strategies for both databases are shown in Additional file 1: Appendix 1.

Search was performed according to the above strategy to obtain the titles of relevant articles. Subsequently, the search strategy was adjusted based on the number of articles obtained after the preliminary search. Articles obtained in this manner were further screened. For systemic reviews related to this topic, their bibliographies were searched to identify potential articles.

All included articles were reviewed by two independent reviewers (Xia L and Fang CY). All disagreements were settled through discussion. If the disagreements could not be settled, a third party was invited to make a final decision. The eligible criteria were as follows: (1) Patients with low-grade gliomas diagnosed by pathology; (2) Adult patients with lesions in the supratentorial region; (3) Trials discussing the relationship between the extent of resection (GTR, STR or biopsy) and prognosis (OS or PFS); (4) 5-year or 1-year OS data were available or could be calculated from other results such as survival plots; (5) If the included cases overlapped, the trial with a greater number of cases would be included. Exclusion criteria were as follows: (1) Patients were pathologically diagnosed as high-grade gliomas in most of the cases included in the article; (2) Patients with pediatric gliomas or subtentorial gliomas; (3) The extent of resection was expressed as percentages rather than GTR, STR and biopsy; (4) 5-year or 10-year survival data were not available.

\section{Data extraction}

Low-grade gliomas are associated with a better prognosis and only a few studies have been focused on the 1-year or 2 -year survival of patients with low-grade gliomas. The literature search also yielded a limited number of studies covering this topic. Therefore, the topic in the search was changed to the association between the extent of resection and 5-year and 10-year OS of patients with low-grade gliomas. Data extraction was performed by two independent reviewers, and the data included the name of the first author, publication time, country, sample size, patients' age, tumor type, the extent of resection, 5-year or 10-year OS and the duration of follow-up. The extent of resection was divided into three categories, i.e., GTR, STR and BX. STR included both subtotal resection and partial resection. If the survival rate was not mentioned when endpoints were reached, the survival rate was calculated from the KaplanMeier curve. Already mentioned that disagreements were settled with discussion.

\section{Quality assessment of primary studies}

The quality of each article was assessed using American Academy of Neurology level of evidence criteria by a research team with four members. All included articles were scored independently by four members and then the sum score was obtained. Any disagreement was settled through discussion until a consensus was reached. The included articles were classified into level I-IV. Among them, Level I indicated the best quality while level IV indicated the lowest credibility.

After data sorting and meta-analysis, the credibility of evidence was assessed using the GRADE system. There were four levels of credibility, i.e., high, moderate, low and very low. A high quality was assigned if the outcome assessment could be altered by further studies; a moderate quality was assigned if the credibility of the outcome 
assessment and the outcome assessment itself might be altered by further studies; a moderate quality was assigned if the credibility of the outcome assessment and the outcome assessment itself might be altered by further studies; a very low quality was assigned if any outcome assessment was uncertain.

\section{Statistical analysis}

Revman5.3 software provided by Cochrane collaboration was employed. Depending on the forest plot and results from the tests of heterogeneity, a fixed effects model or a random effects model was chosen. The relationships among categorical variables were analyzed using an odds ratio (OR) and a95\% confidence interval (CI). Significance was established using CIs at a level of $95 \%$ or $P<$ 0.05.Logarithmicdata were processed by weighting on the basis of sample size. That is, the greater the sample size, the greater the weight was assigned. Funnel plot was used to detect the publication bias.

\section{Results}

\section{Literature search}

According to the search strategy, a total of 1230 English articles (Fig. 1) were eligible. After reviewing the titles, abstracts and full texts, 1210 articles were excluded. Finally, 20 articles involving 2128 cases were included for the meta-analysis (one was a randomized and controlled trial (RCT) and 19 were retrospective studies).

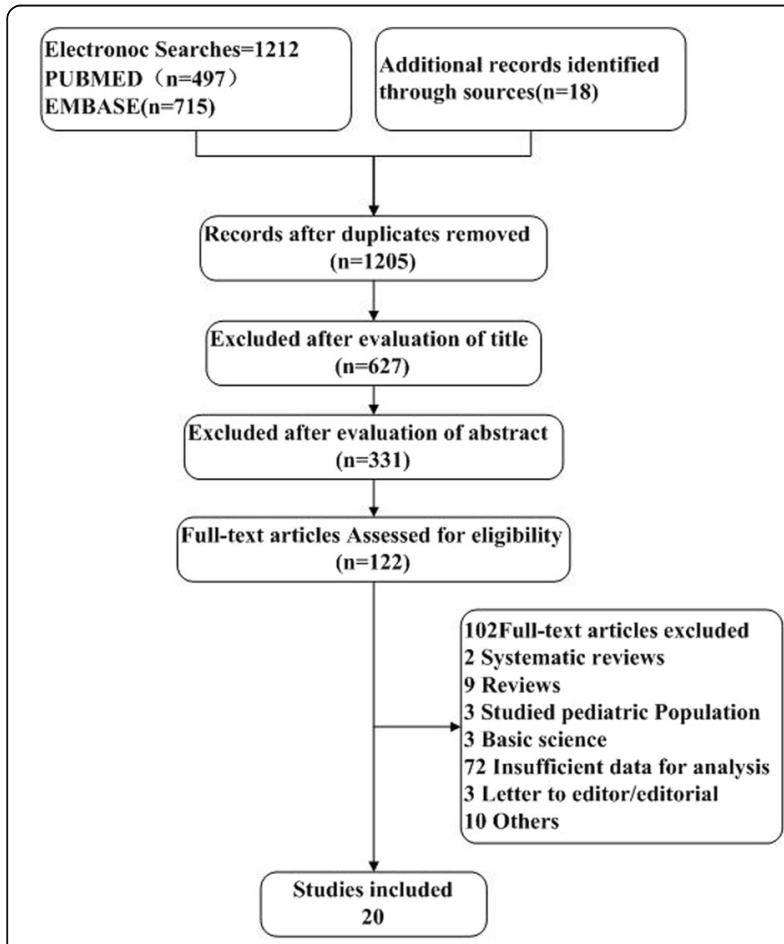

Fig. 1 Flowchart of study selection

\section{Article quality assessment}

None of the included articles was a class Level I study. There were 4 [9-12] class Level II studies, 13 [13-25] class Level III studies and 3 [26-28] class Level IV studies (Table 1). Only 1 study involved a prospective RCT.

\section{Publication bias}

A funnel plot was used to detect the bias in the above articles (Fig. 1). The data points were all located inside the inverted funnel, indicating a small publication bias.

\section{Quality for the body of evidence (GRADE rating)}

The GRADE rating was performed to assess the quality of evidence in terms of the OS outcome and it was found that the quality was of a moderate level. The quality of evidence in class 2 studies was also moderate. The quality of evidence in other studies was low.

\section{Meta-analyses for five-year survival rates}

Among the 20 included studies, 16 studies (1328 cases) compared the 5-year OS of patients with low-grade gliomas after GTR and STR (Fig. 2). The combined results indicated that, as compared with STR, GTR could significantly increase the 5-year survival of patients with low-grade gliomas (OR, 3.90; 95\%CI,2.79 5.45) and there was nearly no heterogeneity between studies $(P=$ 0.83 ). Nine studies (775 cases) compared the 5 -year survival between GTR and biopsy (Fig. 3). The pooled results indicated that, as compared with biopsy, GTR could significantly increase the 5-year survival of patients with low-grade gliomas (OR, 5.43; 95\% CI,3.57 8.26) and there was nearly no heterogeneity between studies $(P=0.23)$. Eleven studies (1147 cases) compared the 5-year survival of patients with low-grade gliomas between STR group and BX group. The combined results indicated that, as compared with BX, STR significantly increased the 5-year survival (OR,1.75; 95\%CI,1.29 2.37) but the heterogeneity was high (I2 = $65 \%, P=0.001$ )(Fig. 4a).Based on the funnel plot, one study was excluded due to high heterogeneity. Analysis of the remaining 10 studies further proved that STR increased the 5-year survival as compared with biopsy (OR,2.59; 95\%CI, 1.81 3.71; $P<0.01 ; \mathrm{Z}=5.19$ ) (Fig. 4b).

\section{Meta-analyses for ten-year survival rates}

A total of 11 studies (907 cases) compared the 10-year survival of patients with low-grade gliomas after GTR and STR. The combined results indicated that, as compared with STR, patients with GTR had the poor 10-year survi$\operatorname{val}(\mathrm{OR}, 7.91 ; 95 \% \mathrm{CI}, 5.12 \sim 12.22)$ and there was no apparent heterogeneity between studies $(P=0.33)$ (Fig. 5). Five studies (185 cases) compared the 10-year survival between GTR and biopsy in low-grade gliomas. The combined results indicated that, as compared with biopsy, GTR 


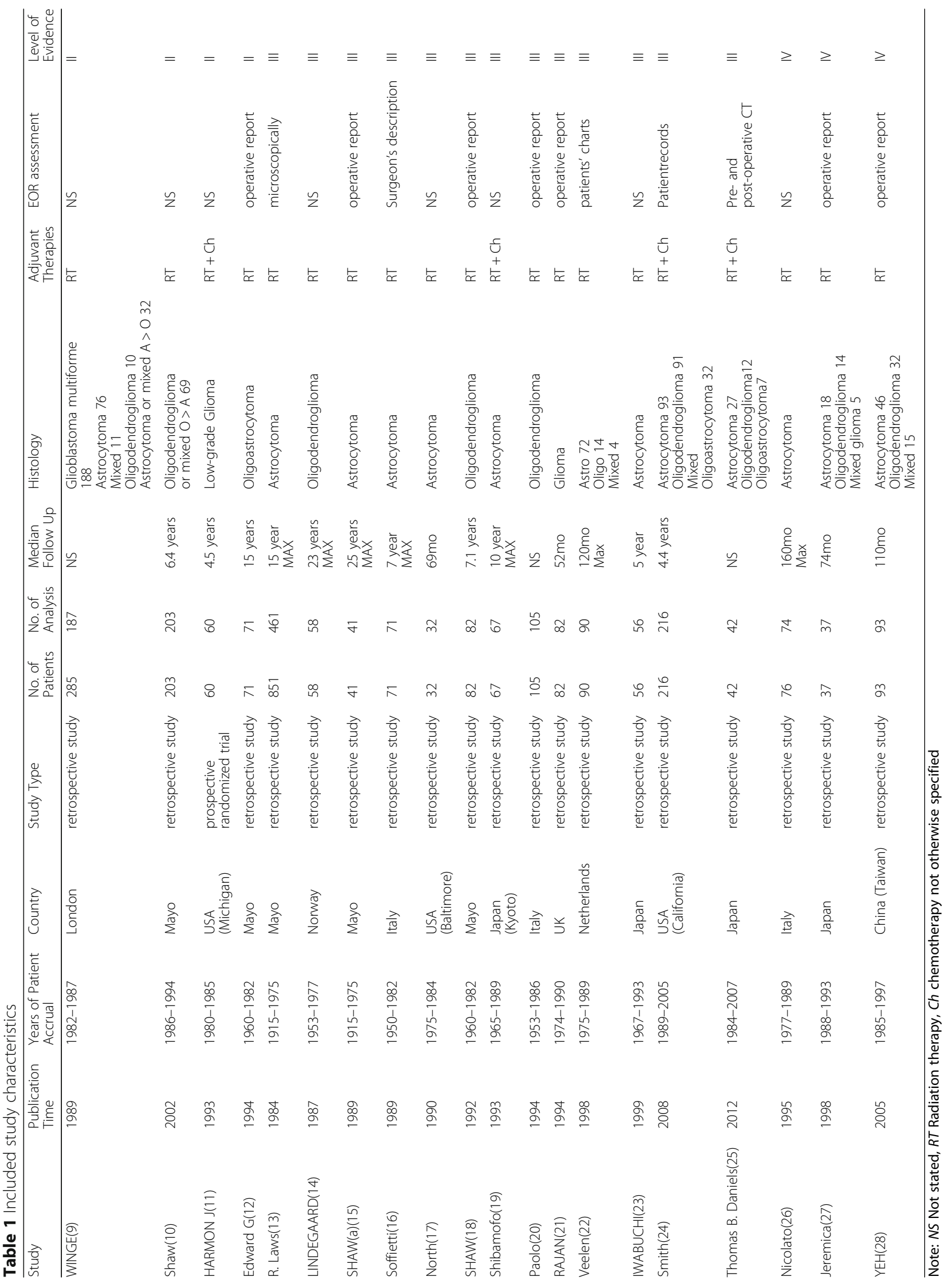




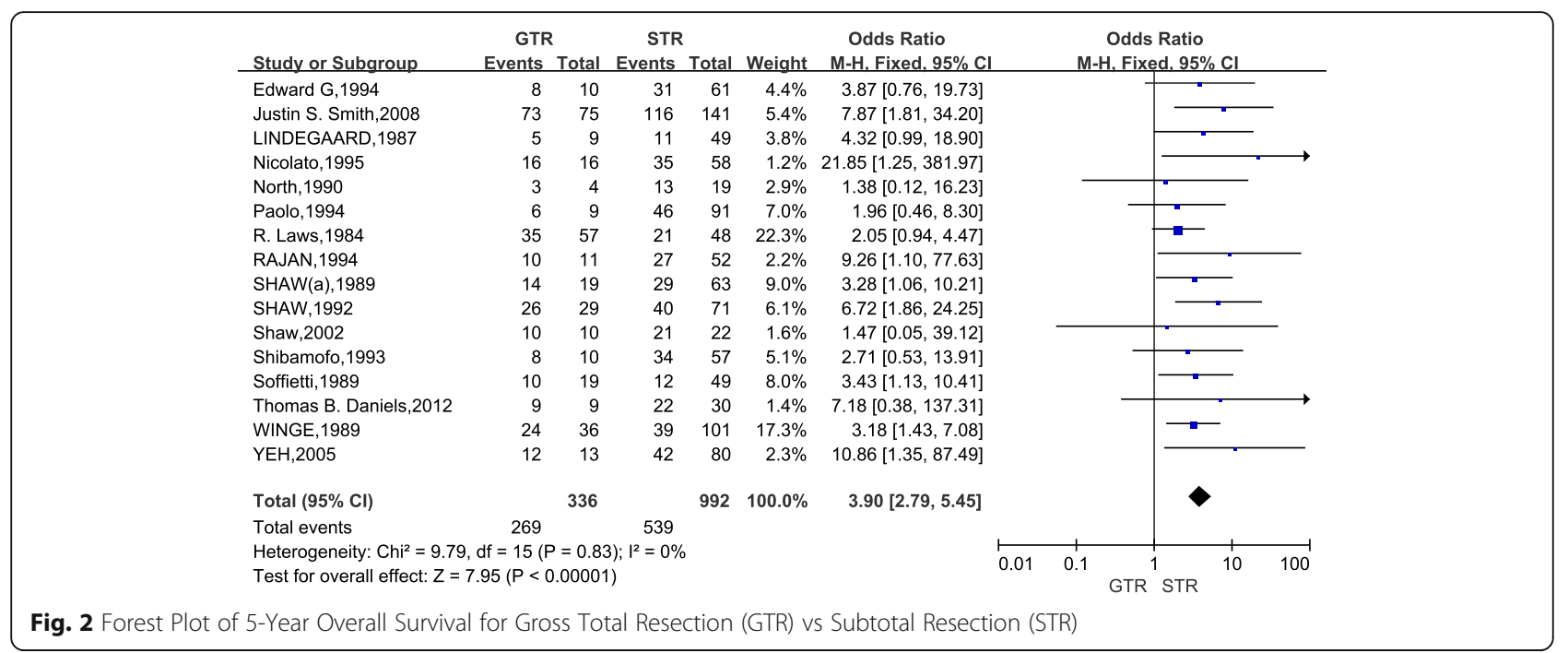

considerably increased the 10-year survival (OR,10.17; 95\% CI,4.02 25.71) and there was no heterogeneity between studies $(P=0.55)$ (Fig. 6). Six studies (408 cases) compared the 10-year survival in low-grade gliomas after STR and biopsy. The combined results indicated that, as compared with biopsy, STR considerably increased the 10-year survival(OR,2.21; 95\%CI,1.16 4.25)and there was also no heterogeneity between studies $(P=0.83)$ (Fig. 7).

\section{Quality for the body of evidence (GRADE rating)}

The GRADE rating was performed to assess the quality of evidence in terms of the OS outcome and it was found that the quality was of a moderate level. The quality of evidence in Level II studies was also moderate. The quality of evidence in other studies was low.

\section{Publication bias}

Funnel plots were used to detect the bias in the above articles (Additional file 2: eFigure S1, Additional file 3: eFigure S2, Additional file 4: eFigure S3, Additional file 5: eFigure S4, Additional file 6: eFigure S5, Additional file 7: eFigure S6). Except studies comparing the 5-year survival of patients with low-grade gliomas between STR group and biopsy group, no publication bias was found in funnel plots, with plots visually symmetrically distributed along the vertical axis.

\section{Discussion}

The clinical value of surgery in low-grade gliomas is heavily disputed [29]. Researchers suggest that although surgery is conducive to pathological diagnosis and remission of symptoms, some low-grade gliomas show infiltrative growth and it is difficult to achieve radical cure through a simple surgery [30]. Low-grade gliomas are generally located in the brain functional areas with obscure boundaries. Surgical resection of low-grade gliomas may lead to dysfunction and impairment of patients' quality of life (QOL) [31]. Some reports showed that the 5-year and 10-year survival of patients receiving GTR was comparable to those receiving STR or no surgery at all [32-34].For this reason, GTR is not the first-line therapy for low-grade gliomas. In recent years, there has been a trend of favoring GTR in the treatment of low-grade gliomas [35, 36].Therefore, we reviewed

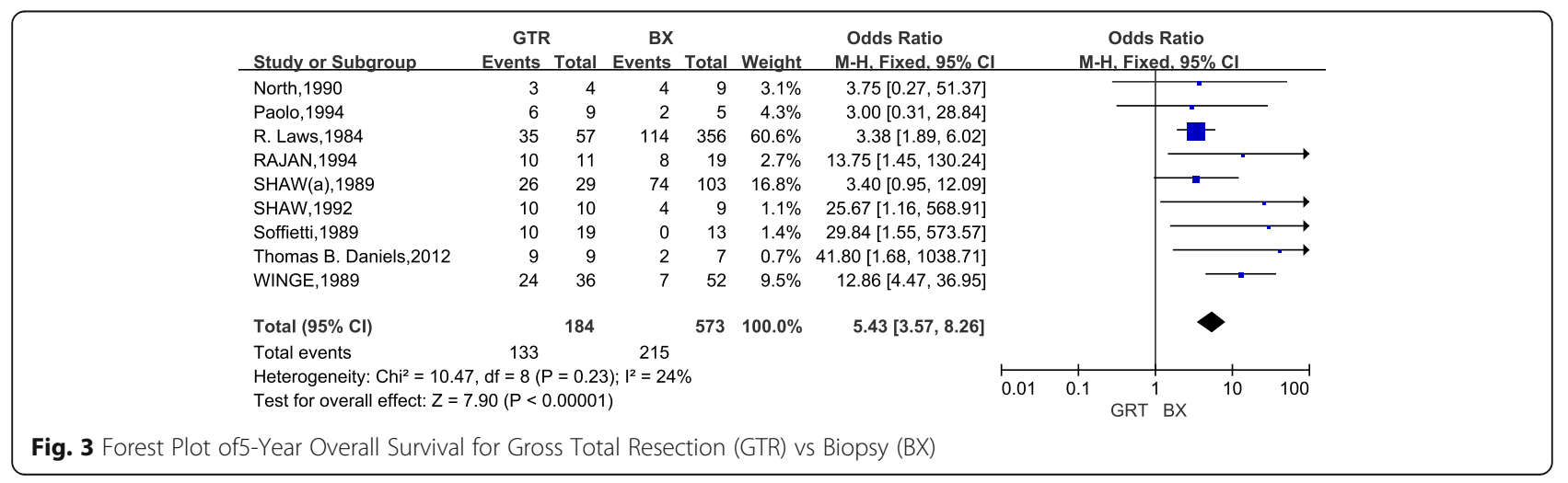




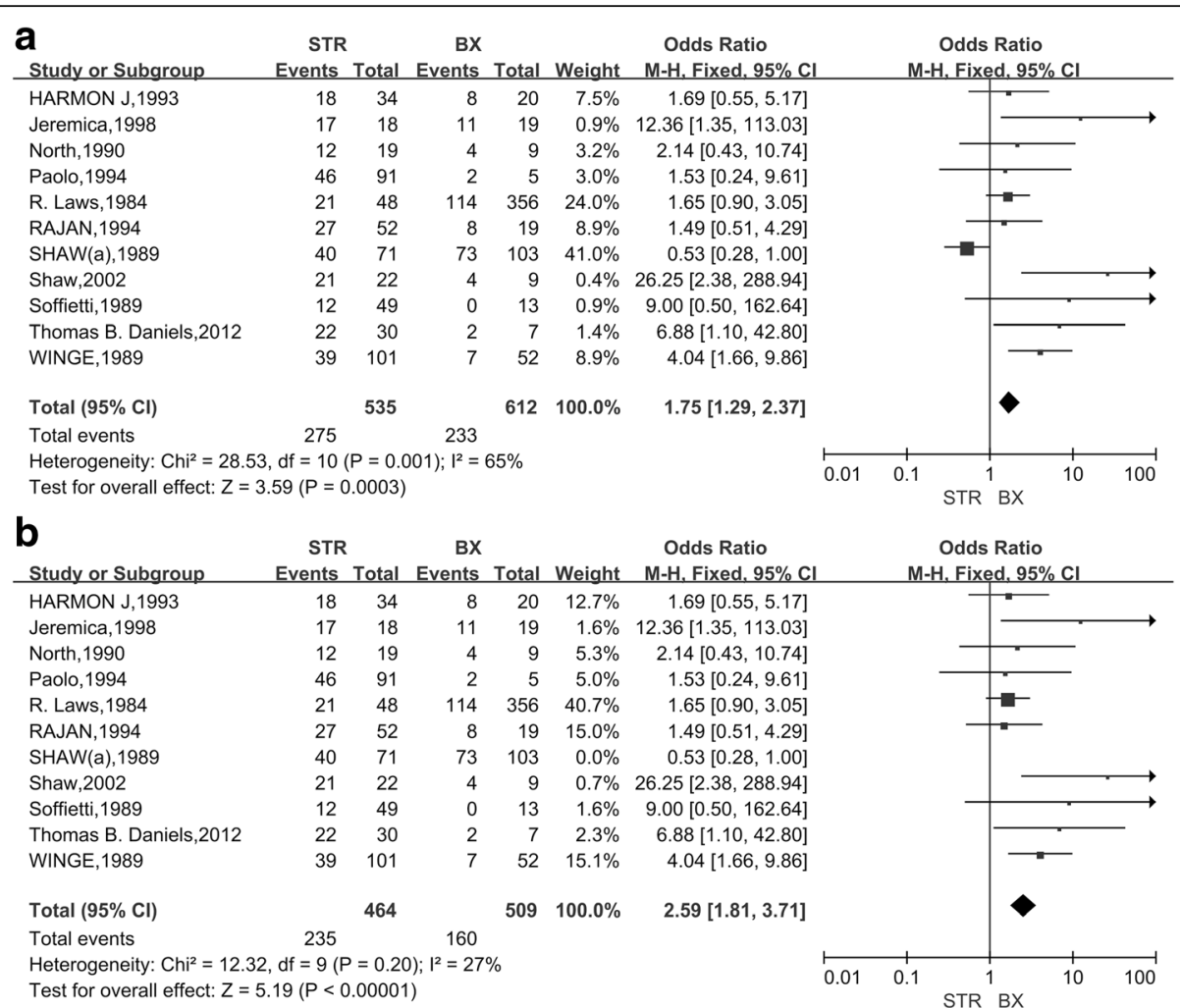

Fig. 4 Forest Plot of 5-Year Overall Survival for Subtotal Resection (STR) vs Biopsy (BX)A. All related studies were included; B. All related studies except one high heterogeneous study were included

relevant studies published up to 2017 and performed a quantitative meta-analysis. The results showed that GTR greatly increased the 5-year and 10-year survival of patients with low-grade gliomas.

Meta-analysis can enhance the credibility of conclusions by using a larger sample size and therefore can resolve the inconsistency among different studies [37]. The findings of meta-analysis are more reliable than those of a single study [38]. Twenty studies focusing on the surgical outcomes of low-grade gliomas were analyzed, as the result showed in the Table 2, patients with
GTR had better prognosis than those with STR and biopsy, similarly, STR is superior to biopsy both in the 5 -year and 10-year OS. Thus, patients with low-grade gliomas are expected to benefit from a greater extent of resection if their safety during the surgery can be ensured.

Better outcome following GTR can be explained by the types of growth that low-grade gliomas exhibit. Firstly, the growth of low-grade gliomas can be divided into three types: confined growth, invasive growth and malignant change. According to recent studies, low-

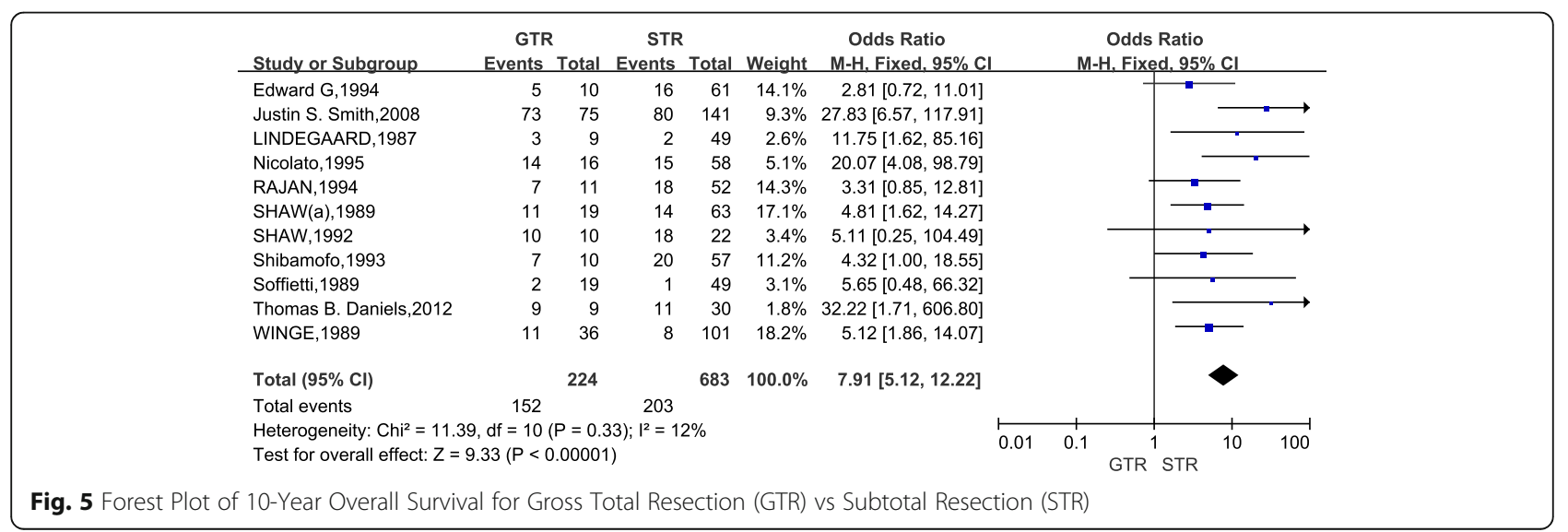




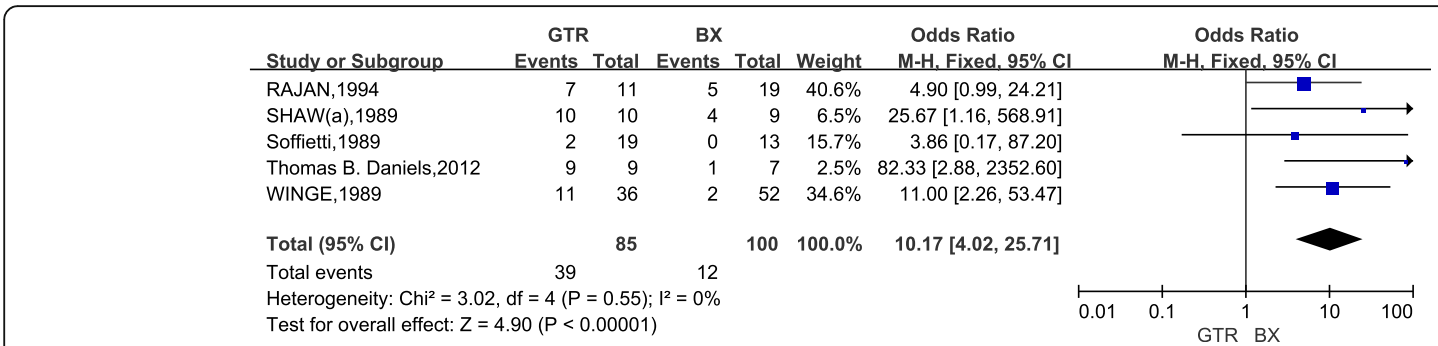

Fig. 6 Forest Plot of 10-Year Overall Survival for Gross Total Resection (GTR) vs Biopsy (BX)

grade gliomas show continuous and slow confined growth before malignant change, resulting in an annual increase of about $4 \mathrm{~mm}$ in size [30]. Invasive growth of low-grade gliomas is demonstrated as the invasion of adjacent white matter tracts, or even the invasion into the contralateral side via corpus callosum. In addition, lowgrade gliomas may evolve into high-grade gliomas [39]. It was reported that $66.4 \%$ of patients with low-grade glioma sunder went de-differentiation within 5 years after surgery, resulting in a worse prognosis [40]. Therefore, early resection of tumor is very important to control infiltration and metastasis. Moreover, the reduction in the tumor load is also conducive to improve effectiveness of subsequent radiochemotherapy. Secondly, from the pathological aspect, histopathology remains the gold standard for the malignancy classification of gliomas. The accuracy of histopathological diagnosis depends on whether a submitted sample is representative [41]. Gliomas are usually associated with heterogeneity in terms of the varying types of cells and different degrees of malignancy in the tumor. That is why the representativeness of the submitted sample is crucial for pathological diagnosis [42]. In one study, a pathological diagnosis based on stereotactic biopsy of astrocytoma was compared with the diagnosis obtained from a surgically resected sample. It was found that sterotactic biopsy underestimated the grades of tumors in $10 \%-25 \%$ of the cases [43]. Therefore, an extensive resection of low-grade gliomas and the submission of all resected specimens for pathological examination can reduce diagnostic errors.
However, some studies might have biases due to limited technical skills and defects in their experimental designs [44]. For example, many researches concerning surgical treatment for low-grade gliomas were retrospective studies. In other studies, the extent of resection was determined based on neurosurgeons' experience or CT scan, which might lead to inconsistent conclusions. Recently, National Cancer Institute (NCI) presented a statistics report on the survival of 2009 patients with low-grade gliomas between 1973 and 2001 [35]. The results showed that surgery prolonged the survival of these patients.

There were other limitations in our study. On the one hand, our meta-analysis included only one prospective and randomized controlled clinical trial while most of the included articles were retrospective studies. There were only four Level II studies included in our analysis while many of the remaining studies were of Level III. However, all results from Level II studies were consistent with our result which favored GTR over STR and biopsy. On the other hand, as mentioned above, low-grade gliomas have a much lower incidence than that of high-grade gliomas, leading to a smaller number of eligible trials in the metaanalysis, so large-scale randomized clinical trials for lowgrade gliomas are urgently needed. Additionally, there were some covariates between studies, such as patients' age, auxiliary treatment methods, tumor size and complications, which could bring some bias to our study.

\section{Conclusion}

Our meta-analysis included only one prospective and randomized controlled clinical trial while most of the

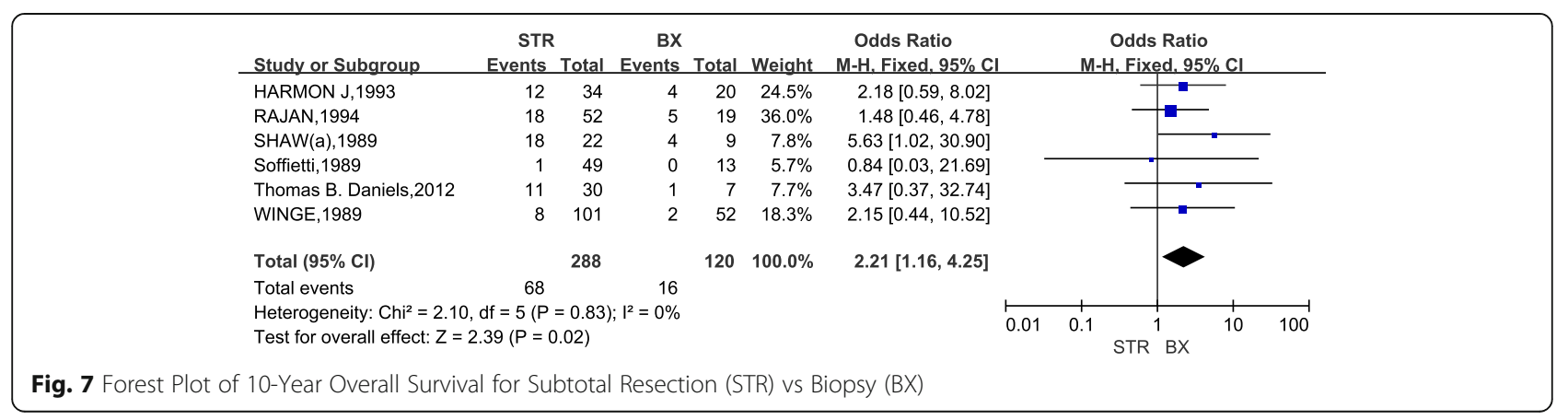


Table 2 Subgroup meta-analysis results

\begin{tabular}{|c|c|c|c|c|c|c|}
\hline Study Factors & NO. of Included Studies & NO. of Patients & Survival Rate & Odds Ratio M-H,Fixed (95\%Cl) & $P$-value & I $^{2}$ statistics, $P$-value \\
\hline \multicolumn{7}{|c|}{ Five-Year Overall Survival } \\
\hline GTR & 16 & 336 & $80.06 \%$ & $3.90[2.79,5.45]$ & $P<0.00001$ & $0 \%, 0.83$ \\
\hline STR & & 992 & $54.33 \%$ & & & \\
\hline GTR & 9 & 184 & $72.28 \%$ & $5.43[3.57,8.26]$ & $P<0.00001$ & $24 \%, 0.21$ \\
\hline$B X$ & & 573 & $37.52 \%$ & & & \\
\hline STR & 10 & 464 & $50.65 \%$ & $2.59[1.81,3.71]$ & $P<0.00001$ & $27 \%, 0.20$ \\
\hline$B X$ & & 509 & $31.43 \%$ & & & \\
\hline \multicolumn{7}{|c|}{ Five-Year Overall Survival } \\
\hline GTR & 11 & 224 & $67.86 \%$ & $7.91[5.12,12.22]$ & $P<0.00001$ & $12 \%, 0.33$ \\
\hline STR & & 683 & $29.72 \%$ & & & \\
\hline GTR & 5 & 85 & $45.88 \%$ & $10.17[4.02,25.71]$ & $P<0.00001$ & $0 \%, 0.55$ \\
\hline$B X$ & & 100 & $12 \%$ & & & \\
\hline STR & 6 & 228 & $29.82 \%$ & $2.21[1.16,4.25]$ & $P=0.020$ & $0 \%, 0.83$ \\
\hline$B X$ & & 120 & $13.33 \%$ & & & \\
\hline
\end{tabular}

included articles were retrospective studies. All evidences were consistent (four class 2 studies) in that they favored GTR over STR and biopsy. There were four class2 studies while many of the remaining studies were of class3. We believe that, as compared with STR and biopsy, GTR could significantly increase the 5-year and 10-year survival of patients with low-grade gliomas. If feasible, GTR is recommended for those patients newly diagnosed as low-grade gliomas. Based on the existing evidences, we believe that more retrospective cohort studies may not provide more useful data. Instead, for low-grade gliomas, high-quality prospective clinical trials are needed to analyze the prognostic factors such as the extent of resection, auxiliary treatment, tumor size, tumor location and complications.

\section{Additional files}

Additional file 1: Supplementary Appendix 1. (DOC 28 kb)

Additional file 2: eFigure S1. Funnel plot for the 5-year mortality for GTR vs STR meta-analysis. The midline of the studies indicates a slight publication bias of studies showing benefit with GTR over STR. (PDF $12 \mathrm{~kb}$ )

Additional file 3: eFigure S2. Funnel plot for the 5-year mortality for GTR vs BX meta-analysis. The midline of the studies indicates a slight publication bias of studies showing benefit with GTR over STR. (PDF 12 kb)

Additional file 4: eFigure S3A. Funnel plot for the 5-year mortality for STR vs BX meta-analysis. The midline of the studies indicates a slight publication bias of studies showing benefit with GTR over STR (All related studies were included). eFigure3B. Funnel plot for the 5-year mortality for STR vs BX meta-analysis. The midline of the studies indicates a slight publication bias of studies showing benefit with GTR over STR (All related studies except one high heterogeneous study were included). (PDF $2433 \mathrm{~kb}$ )

Additional file 5: eFigure S4. Funnel plot for the 10-year mortality for GTR vs STR meta-analysis. The midline of the studies indicates a slight publication bias of studies showing benefit with GTR over STR. (PDF 12 kb)
Additional file 6: eFigure S5. Funnel plot for the 5-year mortality for GTR vs BX meta-analysis. The midline of the studies indicates a slight publication bias of studies showing benefit with GTR over STR. (PDF 12 kb)

Additional file 7: eFigure S6. Funnel plot for the 5-year mortality for STR vs BX meta-analysis. The midline of the studies indicates a slight publication bias of studies showing benefit with GTR over STR. (PDF $12 \mathrm{~kb}$ )

\section{Abbreviations}

BX: Biopsy; Ch: Chemotherapy not otherwise specified; Cl: Confidence interval; GTR: Gross total resection; NCl: National Cancer Institute; NS: Not stated; OR: Odds ratio; OS: Overall survival; PFS: Progression-free survival; QOL: Quality of life; RCT: Randomized and controlled trial; RT: Radiation therapy; STR: Subtotal resection

\section{Acknowledgements}

We thank Dr. Ping Zhang for her help in reviewing the manuscript.

\section{Funding}

The present study was supported by the National Natural Science Foundation of China (Grant No. 81502147), Zhejiang Medical Science and Technology Project $(2,017,194,140,2018$ KY291) and the Youth Scientific Innovation Foundation of Zhejiang Cancer Hospital (Grant No. QN201402).

\section{Availability of data and materials}

Not applicable.

The study is a systematic meta-analysis, all of the data were related to the studies listed in in the Table 1.

\section{Authors' contributions}

LX and CF electronic and manual searches. LX, CF and CS independently inspected all candidate articles and conducted study selection. LX and GC conducted data extraction. LX and GC participated in the design of the study and performed the statistical analysis. GC conceived of the study, and participated in its design and coordination and the drafted the manuscript. All authors read and approved the final manuscript.

\section{Competing interests}

All the authors declare that they have no conflict of interests.

Ethics approval and consent to participate

Not applicable.

Because our study is a systematic meta-analysis, the ethics approval and consent to participate is not relevant to my article type. 


\section{Consent for publication}

Not applicable.

There are no details on individuals reported within the manuscript, so we don't have the consent for publication.

\section{Publisher's Note}

Springer Nature remains neutral with regard to jurisdictional claims in published maps and institutional affiliations.

\section{Author details}

'Department of Neurosurgery, Zhejiang Cancer Hospital, 1 ban shan east Road, Hangzhou, Zhejiang Province 310022, China. ${ }^{2}$ Department of Neurosurgery, The second affiliated hospital of Zhejiang University, Hangzhou, Zhejiang Province 310000, China. ${ }^{3}$ Zhejiang Cancer Hospital, Zhejiang Chinese medical university, Hangzhou, Zhejiang Province 210022, China.

Received: 26 August 2017 Accepted: 13 December 2017

Published online: 06 January 2018

\section{References}

1. Shields LB, Choucair AK. Management of low-grade gliomas: a review of patient-perceived quality of life and neurocognitive outcome. World neurosurgery. 2014;82(1-2):e299-309.

2. Ostrom QT, Bauchet $L$, Davis FG, Deltour I, Fisher JL, Langer CE, Pekmezci M, Schwartzbaum JA, Turner MC, Walsh KM, et al. The epidemiology of glioma in adults: a "state of the science" review. Neuro-Oncology. 2014;16(7):896-913.

3. Morgan LL. The epidemiology of glioma in adults: a "state of the science" review. Neuro-Oncology. 2015;17(4):623-4

4. Weller M, van den Bent M, Hopkins K, Tonn JC, Stupp R, Falini A, CohenJonathan-Moyal E, Frappaz D, Henriksson R, Balana C, et al. EANO guideline for the diagnosis and treatment of anaplastic gliomas and glioblastoma. Lancet Oncol. 2014;15(9):e395-403.

5. Sabha N, Knobbe CB, Maganti M, Al Omar S, Bernstein M, Cairns R, Cako B, von Deimling A, Capper D, Mak TW, et al. Analysis of IDH mutation, 1p/19q deletion, and PTEN loss delineates prognosis in clinical low-grade diffuse gliomas. Neuro-Oncology. 2014;16(7):914-23.

6. Horowitz PM, Chi J. Adult low-grade gliomas: surgery vs biopsy? Neurosurgery. 2013;72(2):N19.

7. Mert A, Kiesel B, Wohrer A, Martinez-Moreno M, Minchev G, Furtner J, Knosp E, Wolfsberger S, Widhalm G. Introduction of a standardized multimodality image protocol for navigation-guided surgery of suspected low-grade gliomas. Neurosurg Focus. 2015:38(1):E4.

8. Brown TJ, Brennan MC, Li M, Church EW, Brandmeir NJ, Rakszawski KL, Patel AS, Rizk EB, Suki D, Sawaya R, et al. Association of the Extent of resection with survival in Glioblastoma: a systematic review and meta-analysis. JAMA Oncol. 2016:2(11):1460-9.

9. Winger MJ, Macdonald DR, Cairncross JG. Supratentorial anaplastic gliomas in adults. The prognostic importance of extent of resection and prior lowgrade glioma. J Neurosurg. 1989;71(4):487-93.

10. Shaw E, Arusell R, Scheithauer B, O'Fallon J, O'Neill B, Dinapoli R, Nelson D, Earle J, Jones $C$, Cascino $T$, et al. Prospective randomized trial of low- versus high-dose radiation therapy in adults with supratentorial low-grade glioma: initial report of a north central cancer treatment group/radiation therapy oncology group/eastern cooperative oncology group study. J Clin Oncol. 2002;20(9):2267-76.

11. Eyre HJ, Crowley JJ, Townsend JJ, Eltringham JR, Morantz RA, Schulman SF, Quagliana JM, al-Sarraf M: A randomized trial of radiotherapy versus radiotherapy plus CCNU for incompletely resected low-grade gliomas: a Southwest Oncology Group study. J Neurosurg 1993, 78(6):909-914.

12. Shaw EG, Scheithauer BW, O'Fallon JR, Davis DH. Mixed oligoastrocytomas: a survival and prognostic factor analysis. Neurosurgery. 1994;34(4):577-82. discussion 582

13. Laws ER Jr, Taylor WF, Clifton MB, Okazaki H. Neurosurgical management of low-grade astrocytoma of the cerebral hemispheres. Neurosurg. 1984;61(4):665-73.

14. Lindegaard KF, Mork SJ, Eide GE, Halvorsen TB, Hatlevoll R, Solgaard T, Dahl O, Ganz J. Statistical analysis of clinicopathological features, radiotherapy, and survival in 170 cases of oligodendroglioma. J Neurosurg. 1987;67(2):224-30.
15. Shaw EG, Scheithauer BW, Gilbertson DT, Nichols DA, Laws ER, Earle JD, Daumas-Duport C, O'Fallon JR, Dinapoli RP. Postoperative radiotherapy of supratentorial low-grade gliomas. Int J Radiat Oncol Biol Phys. 1989; 16(3):663-8.

16. Soffietti R, Chio A, Giordana MT, Vasario E, Schiffer D. Prognostic factors in well-differentiated cerebral astrocytomas in the adult. Neurosurgery. 1989; 24(5):686-92.

17. North CA, North RB, Epstein JA, Piantadosi S, Wharam MD. Low-grade cerebral astrocytomas. Survival and quality of life after radiation therapy. Cancer. 1990;66(1):6-14.

18. Shaw EG, Scheithauer BW, O'Fallon JR, Tazelaar HD, Davis DH Oligodendrogliomas: the Mayo Clinic experience. J Neurosurg. 1992; 76(3):428-34.

19. Shibamoto Y, Kitakabu Y, Takahashi M, Yamashita J, Oda Y, Kikuchi H, Abe M. Supratentorial low-grade astrocytoma. Correlation of computed tomography findings with effect of radiation therapy and prognostic variables. Cancer. 1993;72(1):190-5.

20. Celli P, Nofrone I, Palma L, Cantore G, Fortuna A. Cerebral oligodendroglioma: prognostic factors and life history. Neurosurgery. 1994; 35(6):1018-34. discussion 1034-1015

21. Rajan B, Pickuth D, Ashley S, Traish D, Monro P, Elyan S, Brada M. The management of histologically unverified presumed cerebral gliomas with radiotherapy. Int J Radiat Oncol Biol Phys. 1994;28(2):405-13.

22. van Veelen ML, Avezaat CJ, Kros JM, van Putten W, Vecht C. Supratentorial low grade astrocytoma: prognostic factors, dedifferentiation, and the issue of early versus late surgery. J Neurol Neurosurg Psychiatry. 1998;64(5):581-7.

23. Iwabuchi S, Bishara S, Herbison P, Erasmus A, Samejima H. Prognostic factors for supratentorial low grade astrocytomas in adults. Neurol Med Chir. 1999;39(4):273-9. discussion 279-281

24. Smith JS, Chang EF, Lamborn KR, Chang SM, Prados MD, Cha S, Tihan T, Vandenberg S, McDermott MW, Berger MS. Role of extent of resection in the long-term outcome of low-grade hemispheric gliomas. J Clin Oncol. 2008;26(8):1338-45.

25. Hosoda T, Takeuchi H, Hashimoto N, Kitai R, Arishima H, Kodera T, Higashino Y, Sato K, Kikuta K. Usefulness of intraoperative computed tomography in surgery for low-grade gliomas: a comparative study between two series without and with intraoperative computed tomography. Neurol Med Chir. 2011:51(7):490-5.

26. Nicolato A, Gerosa MA, Fina P, luzzolino P, Giorgiutti F, Bricolo A. Prognostic factors in low-grade supratentorial astrocytomas: a uni-multivariate statistical analysis in 76 surgically treated adult patients. Surg Neurol. 1995;44(3):208-21. discussion 221-203

27. Jeremic B, Shibamoto Y, Grujicic D, Milicic B, Stojanovic M, Nikolic N, Dagovic A. Hyperfractionated radiation therapy for incompletely resected supratentorial low-grade glioma. A phase II study. Radiother Oncol. 1998; 49(1):49-54

28. Yeh SA, Ho JT, Lui CC, Huang YJ, Hsiung CY, Huang EY. Treatment outcomes and prognostic factors in patients with supratentorial low-grade gliomas. Br J Radiol. 2005;78(927):230-5.

29. Forst DA, Nahed BV, Loeffler JS, Batchelor TT. Low-grade gliomas. Oncologist. 2014;19(4):403-13.

30. Jakola AS, Skjulsvik AJ, Myrmel KS, Sjavik K, Unsgard G, Torp SH, Aaberg K, Berg T, Dai HY, Johnsen K, et al. Surgical resection versus watchful waiting in low-grade gliomas. Ann Oncol. 2017;28:1942.

31. Vassal M, Charroud C, Deverdun J, Le Bars E, Molino F, Bonnetblanc F, Boyer A, Dutta A, Herbet G, Moritz-Gasser S, et al. Recovery of functional connectivity of the sensorimotor network after surgery for diffuse low-grade gliomas involving the supplementary motor area. J Neurosurg. 2017;126(4):1181-90.

32. Piepmeier JM. Observations on the current treatment of low-grade astrocytic tumors of the cerebral hemispheres. J Neurosurg. 1987;67(2):177-81.

33. Whitton AC, Bloom HJ. Low grade glioma of the cerebral hemispheres in adults: a retrospective analysis of 88 cases. Int J Radiat Oncol Biol Phys. 1990;18(4):783-6

34. Johannesen TB, Langmark F, Lote K. Progress in long-term survival in adult patients with supratentorial low-grade gliomas: a population-based study of 993 patients in whom tumors were diagnosed between 1970 and 1993. J Neurosurg. 2003:99(5):854-62.

35. Claus EB, Black PM. Survival rates and patterns of care for patients diagnosed with supratentorial low-grade gliomas: data from the SEER program, 1973-2001. Cancer. 2006;106(6):1358-63. 
36. Jakola AS, Myrmel KS, Kloster R, Torp SH, Lindal S, Unsgard G, Solheim O. Comparison of a strategy favoring early surgical resection vs a strategy favoring watchful waiting in low-grade gliomas. JAMA. 2012;308(18):1881-8.

37. Jin ZC, Zhou XH, He J. Statistical methods for dealing with publication bias in meta-analysis. Stat Med. 2015;34(2):343-60.

38. Peterman RM. Statistical power of methods of meta-analysis. Trends Ecol Evol. 1995;10(11):460.

39. Ius T, Pauletto G, Cesselli D, Isola M, Turella L, Budai R, DeMaglio G, Eleopra $R$, Fadiga L, Lettieri $C$, et al. Second surgery in insular low-grade Gliomas. Biomed Res Int. 2015;2015:497610.

40. Gousias K, Schramm J, Simon M. Extent of resection and survival in supratentorial infiltrative low-grade gliomas: analysis of and adjustment for treatment bias. Acta Neurochir. 2014;156(2):327-37.

41. Olson JJ, Kalkanis SN, Ryken TC. Evidence-based clinical practice parameter guidelines for the treatment of adults with diffuse low grade glioma: introduction and methods. J Neuro-Oncol. 2015;125(3):449-56.

42. Hoffman LM, DeWire M, Ryall S, Buczkowicz P, Leach J, Miles L, Ramani AK, Brudno M, Kumar SS, Drissi R, et al. Erratum: spatial genomic heterogeneity in diffuse intrinsic pontine and midline high-grade glioma: implications for diagnostic biopsy and targeted therapeutics. Acta Neuropathol Commun. 2016:4:13.

43. Reardon DA, Wen PY. Glioma in 2014: unravelling tumour heterogeneity-implications for therapy. Nat Rev Clin Oncol. 2015; 12(2):69-70.

44. Ke C, Tran K, Chen Y, Di Donato AT, Yu L, Hu Y, Linskey ME, Wang PH, Limoli $\mathrm{CL}$, Zhou YH. Linking differential radiation responses to glioma heterogeneity. Oncotarget. 2014;5(6):1657-65.

\section{Submit your next manuscript to BioMed Central and we will help you at every step:}

- We accept pre-submission inquiries

- Our selector tool helps you to find the most relevant journal

- We provide round the clock customer support

- Convenient online submission

- Thorough peer review

- Inclusion in PubMed and all major indexing services

- Maximum visibility for your research

Submit your manuscript at www.biomedcentral.com/submit 